\title{
Application of CNN in the Prediction of Hail Cloudy
}

\author{
Jin Lei Cui ${ }^{1, a^{*}}$, Guo Dong $\mathrm{Li}^{2, \mathrm{~b}}$ and Wen Xia Xu ${ }^{3, \mathrm{c}}$ \\ ${ }^{1}$ Department of Applied Mathematics, Xinjiang University of Finance and Economics, P.R. China, \\ 830012 \\ ${ }^{2}$ Department of Applied Mathematics, Xinjiang University of Finance and Economics, P.R. China, \\ 830012 \\ ${ }^{3}$ Xinjiang Weather Modification Office, Urumqi, Xinjiang, P.R. China, 830002

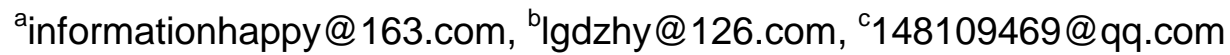

Keywords: Cellular Neural Network; Wavelet transform; Prediction; Life feature vector matrix

\begin{abstract}
This paper studies on the prediction of the hail. First, Cellular Neural Network method acts on the cloud radar images to extract their edge, The cloud's contour feature would be more clear; and then, the edge detection would be processed by wavelet transform. Five different coefficients would be found; At last we construct hail cloud life feature vector matrix, explain the problem by matrix form, so as to find the corresponding rules through the five coefficients, after seeking to rules, through the simulation experiment, to achieve the purpose of hail forecast.
\end{abstract}

\section{Introduction}

Hail is a kind of serious natural disasters. The safety of people's life and property should be threatened by the hail the. So the prediction and identification of hail cloud is particularly critical $^{[1-3]}$. According to the meteorological department, it is mainly based on the analysis of charts, satellite images and radar monitor weather, to judge whether there is a hail; weather radar, satellite, computer, communications and other advanced equipment are widely used in meteorology, greatly improving the tracking ability of hail events. This paper will use the method of image processing of Cellular Neural Network with wavelet transform, to establish the mathematical model, the realization of data mining and analysis of radar image, showing a more ideal rule, so as to realize the identification and prediction of hail cloud ${ }^{[4-5]}$.

\section{Edge detection cell nerve network}

Cellular Neural Network $(\mathrm{CNN})$ is a strong nonlinear simulation systems, the form of cellular neural network is also in constant increase, widely application field. A standard CNN architecture consists of an $\mathrm{M} \times \mathrm{N}$ rectangular array by cells $C(i, j)$ with Cartesian coordinates $(i, j)$, $i=1,2, \cdots, M, j=1,2, \cdots, N \cdot{ }^{[6-10]}$ The sphere of influence $S_{r}(i, j)$, the radius $r$ of cell $C(i, j)$ is defined to be the set of all the neighborhood cells satisfying the following property. $C(k, l) \in S_{r}(i, j)$.

$S(i, j)=\{C(k, l) \mid \max \{k-i|| l-j \mid\} \leq r\} 1 \leq k \leq M,, 1 \leq l \leq N$

For $r$ is a positive integer. The network can sign as equation:

$\frac{d x}{d t}=-x_{i, j}+\sum A(i, j ; k, l) y_{k l}+\sum B(i, j ; k, l) u_{k l}+z_{i, j}$

Where $x_{i, j}, y_{i, j}, u_{i, j} z_{i, j}$ are called state, output, input and threshold of cell $C(i, j)$ 
respectively. $A(i, j ; k, l)$ and $B(i, j ; k, l)$ are called the feedback.

So the standard template of Edge gray $\mathrm{CNN}$ has the form as equation:

$$
\frac{d x}{d t}=-x_{i, j}+\sum_{k=-r}^{r} \sum_{k=-r}^{r} a_{k, l} y_{i+k, j+l}+\sum_{k=-r}^{r} \sum_{k=-r}^{r} b_{k, l} u_{i+k, j+l}+z_{i, j}
$$

The output:

$$
y_{i+k, j+l}=\frac{1}{2}\left(\left|x_{i+k, j+l}+1\right|-\left|x_{i+k, j+l}-1\right|\right)
$$

$(i+k, j+l) \in[1, M] \times[1, N] \quad i=1,2, \cdots M ; j=1,2, \cdots N \quad$ Where $a_{k, l}$ is the parameter of the A-template, $b_{k . l}$ is the parameter of the B-template.

Now we use the special Situation. Let $a_{k, l}, \quad b_{k . l}$ as following in equation :

$$
A=\left[\begin{array}{lll}
0 & 0 & 0 \\
0 & 2 & 0 \\
0 & 0 & 0
\end{array}\right], B=\left[\begin{array}{ccc}
-1 & -1 & -1 \\
-1 & 8 & -1 \\
-1 & -1 & -1
\end{array}\right], Z=-0.5
$$

\section{Wavelet Transform theory}

We use the two-dimensional wavelet transform to process images. $f_{j+1}(x, y) \in L^{2}\left(R^{2}\right), \quad V_{j}^{2}(j \in Z)$ is a separable and resolution analysis of $L^{2}\left(R^{2}\right) \cdot \phi(x, y)=\phi(x) \phi(y)$ is the corresponding of two dimensional scaling function. $\psi(x)$ is the corresponding of one dimensional orthonormal wavelet scale function. If we definite 3 "two-dimensional wavelet"(5):

$$
\left\{\begin{array} { l } 
{ \psi ^ { H } ( x , y ) = \phi ( x ) \psi ( y ) } \\
{ \psi ^ { V } ( x , y ) = \psi ( x ) \phi ( y ) } \\
{ \psi ^ { D } ( x , y ) = \psi ( x ) \psi ( y ) }
\end{array} \quad \left\{\begin{array}{l}
c_{k, m}^{j}=\sum_{l, n} h_{l-2 k} h_{n-2 m} c_{l, n}^{j+1} \\
d_{k, m}^{j, 1}=\sum_{l, n} h_{l-2 k} g_{n-2 m} c_{l, n}^{j+1} \\
d_{k, m}^{j, 2}=\sum_{l, n} g_{l-2 k} h_{n-2 m} c_{l, n}^{j+n} \\
d_{k, m}^{j, 3}=\sum_{l, n}^{l, n} g_{l-2 k} g_{n-2 m} c_{l, n}^{j+1}
\end{array}\right.\right.
$$

These are the standard orthogonal basis in $L^{2}\left(R^{2}\right)$, is composed of one dimensional orthogonal multiresolution analysis in the paper can know:

$$
f_{j+1}(x, y)=\sum_{k, m} c_{k, m}^{j+1} \varphi_{j, k, m}=\sum_{k, m} c_{k, m}^{j} \varphi_{j, k, m}+\sum_{k, m} d_{k, m}^{j, 1} \psi_{j, k, m}^{H}+\sum_{k, m} d_{k, m}^{j, 2} \psi_{j, k, m}^{V}+\sum_{k, m} d_{k, m}^{j, 3} \psi_{j, k, m}^{D}
$$

Using the orthogonal scaling function and wavelet function's decomposition algorithm(6):

The reconstruction algorithm:

$c_{k, m}^{j+1}=\sum_{l, n} h_{k-2 l} h_{m-2 n} c_{l, n}^{j}+\sum_{l, n} h_{k-2 l} g_{m-2 n} d_{l, n}^{j, 1}+\sum_{l, n} g_{k-2 l} h_{m-2 n} d_{l, n}^{j, 2}+\sum_{l, n} g_{k-2 l} g_{m-2 n} d_{l, n}^{j, 3}$

So we can get five coefficients: approximate detail coefficients, level detail coefficients, vertical detail coefficients, diagonal detail coefficients, reconstructed coefficients

\section{Model establishment and analysis}

Construction of hail cloud life feature vector. 8 weathers has be select, hail in 4 , non hail in 4 , average 15 images in every weather for this experiment research. This experiment adopts the MATLAB software programming, the realization of image processing on the clouds. The method of images edge detection using Cellular Neural Network, using the template parameters as the 
processing conditions of Cellular Neural Network, and the function in equation, the theoretical knowledge and basic algorithm of Cellular Neural Network combined with software programming, Cellular Neural Network is to obtain the stable point, thus complete the edge of images processing. RGB image has three layers, different cloud images of a layer of this article only takes the different weather as an illustration, the following images for example, the image below:

\section{Original images}
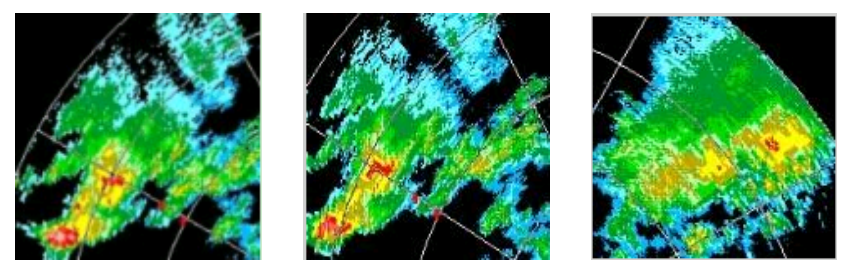

Edge detection cell nerve net
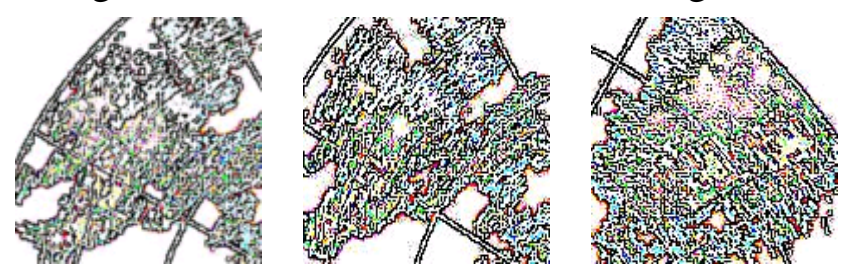

Figure 3. The representative figure of image processing

Images edge detection processing of wavelet transform, the realization of image data mining. According to the filter's point of view, four coefficients namely approximate the detail coefficients, level of detail coefficients, vertical detail coefficients, diagonal detail coefficients will be shown.

Since each image with wavelet transform, there will are five coefficients, due to numerical some coefficient is relatively large. When mining data and programming, will dig out five coefficients are doing $\operatorname{std}\left(\operatorname{std}\left(x_{k}\right)\right)$ transform. $k=1,2,3,4,5$. Each coefficient such that each weather will average about 15 new numerical numbers, setting up $n$ for each weather's the number of images, $a_{i}, b_{i}, c_{i}, d_{i}, e_{i}$ represent respectively the coefficient approximate detail coefficients, level of detail coefficients, vertical detail coefficients, diagonal detail coefficients and the reconstruction of each image. $A=\left(a_{1}, a_{2}, \cdots a_{n}\right)^{T} B=\left(b_{1}, b_{2}, \cdots b_{n}\right)^{T} C=\left(c_{1}, c_{2}, \cdots c_{n}\right)^{T} D=\left(d_{1}, d_{2}, \cdots d_{n}\right)^{T}$

$E=\left(e_{1}, e_{2}, \cdots e_{n}\right)^{T}$ represents approximate detail coefficient matrix, the level of detail coefficients matrix,vertical detail coefficients matrix,diagonal detail coefficients matrix, reconstructed coefficients matrix remodeling of the first weather. So every weather coefficient matrix use the same way to do.

Taking each of weather and each coefficient of the median, we used that life feature vector to find the law of hail cloud. Make(7)

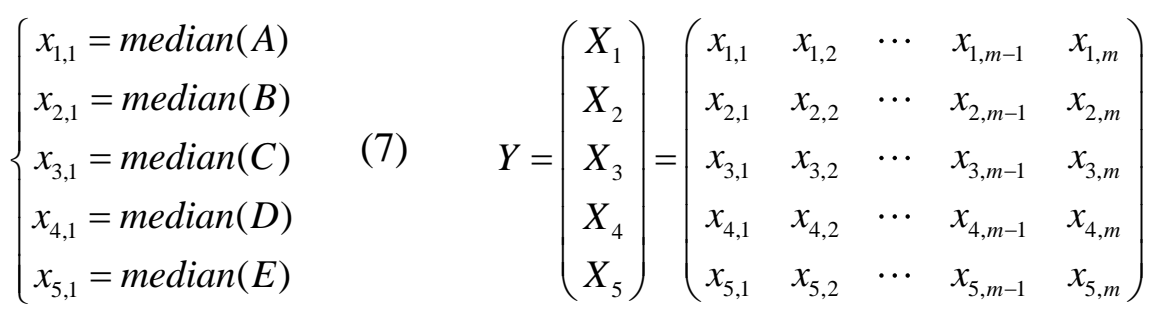

We make each of weather as a unit, the matrix $X_{1}, X_{2}, X_{3}, X_{4}, X_{5}$, to construct the feature vector, in accordance with the five coefficients to form feature vectors, have(8)

Where, $m$ represents the number of the weather, $x_{i j}$ represents the coefficients $i$ of the weather $j\left(j \leq m, i \leq 5, i, j\right.$ are greater than 0 and is an integer), $X_{1}$ represents the approximate detail coefficient vector, $X_{2}$ represents the level of detail coefficients vector, $X_{3}$ represents the 
vertical detail coefficients vector, $X_{4}$ represents the diagonal detail coefficients vector, $X_{5}$ represents reconstructed coefficients vector.

Each coefficient matrix of each image is taken by $\operatorname{std}\left(\operatorname{std}\left(x_{k}\right)\right)$,, to process of the data, with a weather as an example, the results are as follows:

$$
\left\{\begin{array}{l}
x_{1,1}=4.8643 \\
x_{2,1}=6.8529 \\
x_{3,1}=8.3189 \\
x_{4,1}=7.8130 \\
x_{5,1}=0.0379
\end{array}\right.
$$

In this way, the 8 weather, including hail cloud in 4 , non hail cloud in 4. So $Y$ means median vector matrix

The observation of this matrix $X_{1}, X_{2}, X_{3}, X_{4}, X_{5}$ inside $Y$, we get such rules, defined as 3.2-1:

(1) When $X_{1}<10$, it is hail; else, when $X_{1}>10$, it is not hail;

(2)Whether it is hail or not, there is no obvious change about $X_{2}$;

(3) when $X_{3}<20$, it is hail; else, when $X_{3}>20$, it is not hail;

(4) when $X_{4}<13$, it is hail; else, when $X_{4}>13$, it is not hail;

(5) when $X_{5}<0.06$, it is hail; else, when $X_{5}>0.06$, it is not hail;

In other words, if it contents the (1) (3) (4) (5) conditions, you can specify whether the hail. Next, use the simulation experiment to illustrate the rationality to find this rule.

\section{Simulation experiment}

Select to another 8 weather (4 hail cloud, 4 non hail cloud) to validate our rules. On the basis of this method, and simulation results will be as:

Observation in turn each row of the matrix $Y^{\prime}$, will find $x_{4,5}$ and $X_{3}^{\prime}$ not quite conform to the rules of 3.2-1, and $X_{3}^{\prime}$ the need to meet the rules weaken a units, namely, $X_{3}^{\prime}<19$ will hail, $X_{3}^{\prime}>19$ not hail; this also exists a number $x_{3,8}$ that does not conform the rules. That is to say $x_{3,8}$ and $x_{4,5}$ do not comply with 3.2-1 rules. Attenuation of equation (3) conditions, the accuracy rate of the hail can reach $100 \%$, the accurate rate of not hail is $90 \%$, we can think the rule of $3.2-1$ is reasonable. 


\section{Conclusions}

In this paper, using cellular neural network and wavelet transform to process the hail cloud images. To find the motion rules of hail cloud characteristics of life. We find in this paper only the horizontal detail coefficient is not obvious, the coefficient of the rest are displayed obvious rules, and when the four coefficients are all satisfied conditions, cloud is hail clouds, four coefficients satisfy at least three condition, cloud is not hail clouds, through the simulation experiment of hail cloud can know the prediction accuracy is very high, it also found a strong rules for hail forecast, provide an ideal method for hail forecast, but we are a part of image processing, has the error and certain limitations, but I hope this prediction rule can realize the hail.

\section{Acknowledgement:}

The work presented is supported by the financial supports given by research outlay item, MOE (Ministry of Education in China) Project of Humanities and Social Sciences (Project No.13YJAZH040), The National Natural Science Fund (No.11461063), National Social Science Fund (No.14BTJ021) and Xinjiang University of Finance \&Economics Graduate students scientific research innovation project (No.cdyjk2014006).

\section{References}

[1] Zhang Chuang, Chi Jiannan , Zhang Chaohui, et al. Novel color edge detector derived from CNN mode. Computer Engineering and Applications,2008,44(21):17-19

[2] Y. Zhou, and Y. Zhu. Identification of a hail cloud clustering method similar to the evolution of research. Journal of Anhui Agri Sci.2007, 35 (30):9637-9642,9750

[3] Bu-Qing CAO, Jian-Xun LIU, Bin Wen, "Currency Characteristic Extraction and Identification Research Based on PCA and BP Neural Network", JCIT: Journal of Convergence Information Technology, Vol. 7, No. 2, pp. $38 \sim 44,2012$

[4]J. Li, and F. Wang, and W. Mai, and H. Wang.Hail forecast with 3-D hailstorm numerical model at eleven cities in hebei province. Meteorological Monthly. VOL.91, NO.9.2005. 48-51

[5]J. Li, and P. Fan. Investigation on early identification and warning of hail clouds. Journal of Nanjing Institute of Meteorology. Vol. 30 No.1 Feb. 2007. 114-11

[6] L. O. Chua and T. Roska, "Cellular Neural Networks and Visual Computing”, Cambridge: Cambridge University Press, 2000.

[7] Li Guodong, Xu Wenxia, Wang Xu, Efficient Hail Cloudy Identification Scheme Base on CNN, Journal of Convergence Information Technology,2012. Vol. 7(22), p 669-676

[8] Guodong Li, Xu Wang. Hail identification analysis from Radar image by CNN. Applied Mechanics and Materials ,2012.Vols. 155. p 841-845.

[9] G. li and L.Min, and H.Zang, "Color edge detections based on Cellular Neural Network" International Journal of Bifurcation and Chaos, Vol. 18, No. 4 (2008) 1231-1242.

[10] Dr. Hanan A.R.AKKAR, "Optimizing Opto-Electronic Cellular Neural Networks Using Bees Swarm Intelligent", IJIPM: International Journal of Information Processing and Management, Vol. 1, No. 1, pp. 114-125, 2010 